\title{
Costa Rica und seine Verfassung vom 7. November 1949 in Vergangenheit und Gegenwart
}

\author{
von Jochen Fuchs
}

\section{Einführung}

Am 7. November 1949 verabschiedete eine verfassungsgebende Versammlung die augenblicklich gültige Verfassung des Landes in ihrer ursprünglichen Form. Sie besteht aus 18 Abschnitten, die insgesamt 197 Artikel umfassen. Als dreizehnte Verfassung seit der Unabhängigkeitserklärung von Spanien im Jahre 1821 zeichnet sie sich mit ihren bisher 47 'Dienstjahren` durch eine für costaricanische Verhältnisse ungewöhnlich hohe Stabilität aus. Lediglich eine ihrer Vorgängerinnen übertrifft sie (noch) in dieser Hinsicht. Sieht man von diesem Umstand ab, dem für die interne Entwicklung ein größeres Gewicht zukommt, als von außenstehenden Beobachtern gemeinhin zugebilligt, so existieren allerdings noch weitere Punkte, die diese Verfassung für uns sowohl unter politischen wie auch unter verfassungsrechtlichen Gesichtpunkten nicht uninteressant erscheinen lassen.

Als erster sei hier die faktische Ergänzung der klassischen Staatsgewalten um den Obersten Wahlgerichtshof als vierte Gewalt genannt. Mit der im Jahre 1975 erfolgten Änderung des Art. 9 Constitución Política erhielt diese Institution diesen Status auch de jure.

Ferner prägt die Verfassung das Bemühen, der mit einem Präsidialsystem verbundenen Machtkonzentration in den Händen der exekutiven Gewalt wirksam Grenzen zu setzen. Ein Mittel hierzu ist das seit 1949 immer restriktiver gefaßte Wiederwahlverbot für den Präsidenten (Art. 132) - das seine Entsprechung in der parlamentarischen Zwangsrotation gemäß Art. 107 findet. Eine weitere diesem Ziel dienende Methode stellt die Ausgliederung von Aufgaben aus dem exekutiven Kompetenzbereich und deren Úbertragung an sogenannte rautonome Einrichtungen' (Art. $188 \mathrm{ff}$.) dar.

Ungewohnt für einen an die abstrakte Unverbindlichkeit eines Art. 20 I GG gewöhnten Betrachters erscheint auch die detaillierte Berücksichtigung nicht nur der bürgerlichen Freiheitsrechte einschließlich (straf)prozessualer Garantien (Art. 20-49 CP), sondern auch ihrer sozialen Pendants in Art. 50-75 CP, unter denen weder Mindestlohn und -urlaub, noch Sozialleistungen und Arbeitslosenhilfe fehlen.

Der im Vergleich allerdings wohl bekannteste Artikel dürfte Art. 12 CP sein, der seit 1949 unverändert das nicht nur für Mittelamerika einzigartige Verbot der Existenz einer Armee oder militärischer Verbände in Friedenszeiten enthält. Seinem Bekanntheitsgrad entspricht seine Umstrittenheit: Während auf der einen Seite Vertreter der Friedensbewegung auch in Europa Costa Rica als leuchtendes Vorbild darstellen, ${ }^{1}$ reden Vertreter der US-amerikanischen Regierung wenn schon nicht der Aufhebung, so doch der

1 Vgl. Bird, Leonard A./Maislinger, Andreas, Costa Rica - Ein Land ohne Armee, Wien 1980. 
Mißachtung dieses Verbotes das Wort. ${ }^{2}$ Andere Untersuchungen kommen zu dem Ergebnis, daß Art. 12 bereits seit langem in der Realität keine Gültigkeit mehr besitze. ${ }^{3}$ Im Zusammenhang mit der am 17. November 1983 vom Präsidenten Monge verkündeten 'ständigen, aktiven und unbewaffneten Neutralität ' Costa Ricas, die bisher allerdings noch nicht in der Verfassung verankert wurde, ergibt sich außerdem das Problem, inwieweit diese Erklärung nicht im Widerspruch zu Art. 12 steht. ${ }^{4}$ Als Grund dafür wird angeführt, daß eine unbewaffnete Neutralität für einen dauernd neutralen Staat unzulässig sei. $^{5}$

Welcher Position man nun den Vorzug geben will, mag an dieser Stelle dahingestellt bleiben. Klargeworden dürfte sein, daß es jedenfalls lohnend erscheint, der costaricanischen Verfassung eine höhere Aufmerksamkeit zu zollen, als man dies für gewöhnlich gegenüber der Verfassung eines von vielen abschätzig zu den 'Bananenrepubliken`gezählten Landes zu tun bereit ist.

\section{Der historische Kontext}

Seit seiner Entdeckung durch Kolumbus 1502 bildete Costa Rica einen Teil des spanischen Imperiums. Während der kolonialen Epoche blieb es als südlichste Provinz des Generalkapitanats Guatemala weitgehend isoliert. Die Mehrzahl der Conquistadoren mieden das an begehrten Edelmetallen arme Land. Mit dem Anbau von Kakao und Tabak mühte sich die zahlenmäßig geringe Bevölkerung bis auf Ausnahmen vergeblich, die wirtschaftlich desolate Lage zu konsolidieren. ${ }^{6}$ Die seit Beginn des 19. Jahrhunderts in Lateinamerika verstärkt anzutreffenden Unabhängigkeitsbewegungen fanden in Costa Rica kaum ein Echo. Die als Antwort darauf von Spanien ausgehenden Reorganisationsbestrebungen, die in der 1812 verabschiedeten 'Constitución de Cádiz' gipfelten, berührten das Land nur am Rande. ${ }^{7}$ Der nach der Vertreibung der Franzosen zurückgekehrte Ferdinand VII gab allerdings einer restaurativen Politik den Verzug und verurteilte das Experiment von Cádiz zum Scheitern. Als daraufhin zunächst Mexiko und dann auch Guatemala und die übrigen zentralamerikanischen Provinzen sich von Spanien lossagten, mochte Costa Rica nicht abseits stehen und tat es ihnen gleich. Am 29. Oktober 1821 erklärte man sich für unabhängig.

Die darauffolgenden zwei Jahrzehnte wurden innenpolitisch geprägt durch den Streit

2 Vgl. etwa: o.A., Quo Vadis, in: Lateinamerika-Nachrichten Jg. 9, Nr. 99, Berlin 1981, S. 32; o.A., Costa Rica rüstet auf, Lateinamerika-Nachrichten Jg. 10, Nr. 109/110, Berlin 1982, S. 64.

3 O.A., La militarización en Costa Rica, INCEP (Hrsg.), Guatemala 1975.

4 Schindler, Dietrich, Die Neutralität Costa Ricas aus Schweizer Sicht, in: Andreas Maislinger, Costa Rica, Innsbruck 1986, 97 ff. (103 f.).

5 Verosta, Stephan, Die dauernde Neutralität, Wien 1967, S. 74.

6 Hall, Carolyn, Costa Rica, San José 1984, S. 134 ff.; vgl. dazu auch: Fernandez, Leon, Historia de Costa Rica durante la dominación espan̂ola 1502-1821, 2. Aufl. San José 1975.

7 Monge Alfaro, Carlos, Historia de Costa Rica, 13. Aufl. San José 1974, S. 152; vgl. dazu auch: Volio, Marina, Costa Rica en las Cortes de Cádiz, San José 1980. 
zwischen dem (liberalen) San José und der ehemaligen Kolonialhauptstadt Cartago um die Vorherrschaft. Außenpolitisch schwankte das Land zwischen einem Anschluß an das mexikanische Reich Iturbides, der Bildung einer zentralamerikanischen Föderation und staatlicher Autonomie. ${ }^{8}$ Mit der Regierungsübernahme durch Carillo (1838) setzten sich die liberalen Kräfte nach mehreren bürgerkriegsähnlichen Auseinandersetzungen durch. Ókonomisch begann der Aufstieg des Kaffees zum maßgeblichen Exportprodukt. Das Gesicht des Landes änderte sich. In den Jahren bis 1870 gaben die patrimonial-oligarchischen Kräfte, allen voran die Kaffeebarone, den Ton an. Außenwirtschaftlich herrschte eine enge Anbindung an England, dem Hauptabnehmerland für costaricanischen Kaffee. ${ }^{9}$

Ein Versuch des nordamerikanischen Abenteurers William Walker, Costa Rica und die anderen mittelamerikanischen Staaten den USA einzuverleiben, scheiterte. Unter Führung des Generals Guardia schlug eine vereinigte zentralamerikanische Streitmacht die Invasionsarmee. Gestützt u. a. auf diesen Erfolg übernahm Guardia in einem Coup d'état die Macht. Seine Politik zielte auf eine Modernisierung des Staates. In seine Amtszeit fällt auch die Ausarbeitung der Verfassung von 1871. Diese - wenn auch mit einigen Unterbrechungen bis 1948 gültige - Verfassung avancierte zur klassischen Konstitution Costa Ricas und bildete die Basis für die Diskussion um die Verabschiedung der Verfassung von 1949.

Zu Guardia's Zeit drängte auch zunehmend ausländisches Kapital ins Land. Keith, Mitbegründer der United Fruit Company, genoß umfangreiche Sonderrechte und verfügte nicht nur über einen bedeutenden Teil der Eisenbahnlinien, sondern auch über riesige Ländereien für das überaus erfolgreiche Bananengeschäft. ${ }^{10}$

Gegen Ende des 19. Jahrhunderts traten erstmals auch in Ansätzen Organisationen der abhängig Beschäftigten auf. Zunächst noch vorwiegend mit einer sowohl gegen die auf Säkularisation bedachten Liberalen ankämpfenden, als auch der christlichen Soziallehre verbundenen Kirche liiert, emanzipierten sich diese im Lauf der Zeit und traten, verstärkt seit den zwanziger Jahren, auch mit eigenen Programmen an die Óffentlichkeit. Während des 1. Weltkrieges kam es aufgrund von Absatzschwierigkeiten auf dem englischen Markt zu einer einschneidenden Krise. Als auch das übliche Kriseninstrumentarium dagegen nichts auszurichten vermag, putscht der Kriegsminister Tinoco mit Unterstützung einer an der Erteilung von Olbohrkonzessionen interessierten nordamerikanischen Firma. Um seine Legitimationsbasis, die sich bisher auf das Versprechen beschränkte, die Probleme des Landes zu lösen, zu erweitern, dekretierte er eine neue Verfassung. Deren Artikel 10 stellt insofern eine erwähnenswerte Neuerung dar, als er dem

8 Ernst, Manfred, Costa Rica, Bonn 1984, S. 28; vgl. dazu auch: Fernandez Guardia, Ricardo, La Independencia, San José 1971.

9 Hiedl, Peter/Rausch, Renate, Costa Rica, in: Dieter Boris u. Renate Rausch (Hrsg.), Zentralamerika, Köln 1983, S. 353; vgl. dazu auch: Hall, Carolyn, El café y el desarrollo histórico-geográfico de Costa Rica, San José 1978 .

10 Bitter, Wilhelm, Die wirtschaftliche Eroberung Mittelamerikas durch den Bananen-Trust, Darmstadt 1974, S. 95; vgl. dazu auch: Stewart, Watt, Keith and Costa Rica, Albuquerque 1964. 
Staat die Verpflichtung auferlegt, für das Wohlergehen der arbeitenden Klassen Sorge zu tragen. ${ }^{11}$ Bereits nach weniger als drei Jahren endete allerdings die einzige Militärdiktatur in Costa Ricas neuerer Geschichte. Die ursprünglich außer Kraft gesetzte Verfassung von $1871 \mathrm{kam}$ wieder zu Ehren. Die wirtschaftliche Talsohle war überwunden und während der zwanziger Jahre konnte das Land eine auf die nur gering diversifizierte Exportpalette gestützte ökonomische Aufwärtsentwicklung verzeichnen, die erst mit dem Ausbruch der Weltwirtschaftskrise ihr Ende fand. Ihr Ende fand mit dieser auch die fast ein halbes Jahrhundert währende Alleinherrschaft der liberalen Oligarchie. Die Macht der zu dieser Zeit sich fast als 4 . Gewalt ‘ im Staate darstellende United Fruit Company blieb gleichfalls nicht unangefochten: 1934 richtete sich der größte Streik in der Geschichte dieses Landes, maßgeblich geleitet von der erst kurz zuvor gegründeten KP, gegen diesen zu den ältesten Multis der Welt zählenden Konzern. ${ }^{12}$

In den dreißiger Jahren wollte man die wirtschaftlichen Probleme mit dem von Keynes entwickelten Instrumentarium lösen. Doch dies zeigte sich als nur bedingt tauglich, das Auseinanderdriften der tradierten Gesellschaftssektoren mehr als nur zu verlangsamen. 1940 übernahm Calderón, ein christlich-sozialer Politiker, gestützt auf die nationalrepublikanische Partei (PRN) und einen überwältigenden Wahlerfolg (86\% der Stimmen) die Präsidentschaft. ${ }^{13}$ Die breite Unterstützung bröckelte jedoch nur allzu rasch, da es ihm nicht gelang, allen, oft gegensätzlichen Interessen der ihn ursprünglich Unterstützenden gerecht zu werden. In den Kongreßwahlen von 1942 verlor die PRN stark, KP und bürgerliche Oppositionsparteien errangen jeweils ca. 20 Prozent der Stimmen.

Calderón suchte nach einem Ausweg aus diesem Dilemma und fand ihn in einem Bündnis mit der KP. Begünstigt wurde dies außenpolitisch durch die Zugehörigkeit Costa Ricas zur Anti-Hitler-Koalition, innenpolitisch durch die auch von der Kirche geforderte Lösung der sozialen Frage. Die PRN ging auf die von der KP gestellte Forderung nach der Aufnahme eines detaillierten Katalogs sozialer Grundrechte in die Verfassung ein. Im Gegenzug benannte sich die KP in PVP (Partei der Avantgarde des Volkes) um und erhielt vom Erzbischof Sanabría eine 'Unbedenklichkeitsbescheinigung` des Inhalts, daß für Katholiken eine Zusammenarbeit mit der PVP erlaubt sei.

Gegen diese 'Volksfront ' kristallisierten sich drei oppositionelle Fraktionen heraus: Eine sozialdemokratisch-panzentralamerikanistische unter maßgeblicher Beteiligung von José Figueres und zwei bürgerliche Gruppen, deren gemäßigtere von Ulate repräsentiert wurde. Die politischen Auseinandersetzungen spitzten sich zunehmend zu und eskalierten nach den Präsidentschaftswahlen von 1948. Die Opposition hatte Ulate ins Rennen geschickt, der nach der ersten Stimmauszählung und dem Urteil eines Gerichts über den von PVP und PRN nominierten Calderón siegte. Das von letzteren beherrschte Par-

11 Peralta, Hernan G., Las constituciones de Costa Rica, Madrid 1962, S. 498 f.; vgl. dazu auch: Cruz, Vladimir de la, Las luchas sociales en Costa Rica 1870-1930, 2. Aufl., San José 1981.

12 Ernst, Manfred, Uberblick in: Manfred Ernst und Sönke Schmidt (Hrsg.), Berlin 1986, S. 21.

13 Creedman, Theodore S., Historical Dictionary of Costa Rica, Metuchen, New York, London 1977, S. 25. 
lament weigerte sich aber, das Ergebnis zu bestätigen, da inzwischen fast alle Wahlzettel verschwunden waren. ${ }^{14}$

José Figueres reagierte darauf mit einem Aufruf zum bewaffneten Kampf gegen die seiner Ansicht nach sich unrechtmäßig an der Macht haltende Regierung. Nach relativ kurzer Dauer des Bürgerkrieges und unter dem Eindruck einer von Somoza (Nicaragua) in Aussicht gestellten Invasion kapitulierten nach der regulären Armee auch die hauptsächlich von der PVP aufgestellten Freiwilligenverbände. Figueres mußte zuvor ihrem Führer Mora allerdings zusichern, daß die Sozialgesetzgebung, wie auch die Existenz der PVP nicht gefährdet würde. ${ }^{15}$ Figueres, der aber nur den ersten Teil des Versprechens hielt, übernahm an der Spitze einer Junta für 18 Monate die Regierungsgewalt. Vereinbarungsgemäß trat er sie danach an den Sieger der 448 er Wahlen, Ulate, ab. Bei Einbeziehung auch der folgenden Wahlen in die Betrachtung stellt sich ein solcher Wechsel zwischen sozialdemokratisch und bürgerlich orientierten Regierungen für Costa Ricas Geschichte als typisch dar.

Außenpolitisch erfolgte eine prinzipielle Annäherung an die USA, im Verhältnis zu den anderen Ländern der Region fühlt sich Costa Rica in einer modellhaften Sonderrolle. Auf wirtschaftspolitischem Sektor strebte man mittels verstärkter staatlicher Interventionen und Lenkungsmaßnahmen eine allgemeine Modernisierung an. Die einseitige Abhängigkeit vom Kaffee und den Bananen sollte aufgebrochen werden und vor allem die mittelständische Wirtschaft gestärkt werden. ${ }^{16}$ Parallel dazu suchte man über eine forcierte Urbarmachung brachliegender Flächen auch den durch die demographische Entwicklung hervorgerufenen Druck aufzufangen. Als flankierende Maßnahme propagierte man die qualitative und quantitative Verbesserung des Bildungsniveaus der Bevölkerung, ${ }^{17}$ was angesichts des seit 1949 geltenden Verzichts auf eine Armee auch nicht an der Finanzierbarkeit scheiterte. (Costa Rica ist sehr stolz darauf, Schulen statt Kasernen zu bauen und Lehrer anstelle von Soldaten ausgebildet zu haben). ${ }^{18}$

1963 trat das Land dem MCCA, dem gemeinsamen Markt Zentralamerikas, bei. Auf diese Weise hoffte man, die auf ökonomischem und sozialem Gebiet bisher errungenen Erfolge zu sichern und auszubauen, um so auch weiterhin der inzwischen usus gewordenen Bezeichnung 'Schweiz Zentralamerikas' gerecht zu werden. Bis weit in die siebziger Jahre hinein schien Costa Rica sich sowohl in wirtschaftlicher wie auch gesellschaftspolitischer Hinsicht als Modellfall und taugliches Vorbild für die anderen lateinamerikani-

14 Shafer, Robert Jones, A History of Latin America, Lexington, Toronto 1978, S. 740 f.; vgl. dazu auch: Aguilar, Bulgarelli, Oscar R., Costa Rica y sus hechos políticos de 1948, San José 1969.

15 Obregon Loria, Rafael, Conflictos militares y políticos de Costa Rica, San José, 1951, vgl. dazu auch: Rojas Bolan̂os, Manuel, Lucha social y guerra civil en Costa Rica, 1940-1948, 3. Aufl., San José 1982.

16 Ernst, Manfred, (vgl. Anm. 8), S. 84 f.

17 Jaksch, Hans Jürgen, Optimale Investitionsvarianten in Costa Rica von 1961-1966, Frankfurt/a.M. 1966, S. 18 f.; vgl. dazu auch: Garcia, José Fernando, Educacion y desarrollo en Costa Rica, in: Revista paraguaya de sociologia, Jg. 14, Nr. 39/40 Asuncion 1977, S. 195 ff.

18 Monge, Luis Alberto, La neutralidad de Costa Rica, San José 1984, S. 60 f.; Montero de Wolff, Isabel, Ein Zukunftstraum wird wahr: das Land ohne Soldaten, in: Lateinamerika, Jg. 2, Bonn 1975, S. 3 ff.

19 Grenz, Wolfgang, Costa Rica als Vorbild, in: Úbersee Rundschau, Hamburg 1968, Jg. 20, Heft 2, S. 15 f. 
schen Staaten zu bewähren. ${ }^{19}$ Erst in den letzten Jahren geriet das Land in den Sog der vor allem Dritte-Welt-Länder treffende weltweiten Wirtschaftskrise. Verstärkt wurden die Auswirkungen noch durch hauptsächlich im Bananensektor auftretende Krankheiten und soziale Konflikte, gepaart mit den sich aus einer hohen Staatsverschuldung ergebenden Schwierigkeiten. Außenpolitisch zeitigen auch die Auseinandersetzungen in den anderen zentralamerikanischen Staaten ihre Auswirkungen, sei es daß Costa Rica vor ein wachsendes Flüchtlingsproblem ${ }^{20}$ gestellt wird, sei es, daß seine nördlichen Regionen von den 'Contras` als Aufmarschgebiet mißbraucht werden. Doch läßt sich das sich anbahnende Scheitern des costaricanischen Weges nicht allein mit exogenen Faktoren befriedigend erklären. ${ }^{21}$ Vielmehr konnte in den vergangenen Jahrzehnten der gesellschaftliche Ausgleich nur auf der Basis einer flächenmäßigen Ausdehnung der Anbauflächen (sog. innere Kolonisation) einerseits und durch die zunehmende Außenverschuldung andererseits gewährleistet werden; als beide Mittel an ihre Grenzen stießen, stieß auch das 'Modell Costa Ricar an diese, was sich aber bisher noch nicht auf die Ebene der Verfassungsentwicklung auswirkte, sieht man von der Unmöglichkeit ab, im Kongreß eine Mehrheit für die Aufnahme der ständigen Neutralität in die Verfassung zu finden.

\section{Die Verfassung von 1949 (Constitución Polítìca de la República de Costa Rica)}

Die von der verfassungsgebenden Versammlung 1949 angenommene Verfassung lehnt sich in zentralen Punkten eng an ihre mit der Machtübernahme der Junta aufgehobene Vorgängerin an. Jene war in ihrer Urfassung 1871 verabschiedet und bis 1948 etliche Male ergänzt und abgeändert worden. Der ursprüngliche Plan der sozialdemokratisch orientierten Junta unter Führung von José Figueres, eine radikale Verfassungsrevision vorzunehmen, ließ sich nicht realisieren. Der zu diesem Zweck bereits ausgearbeitete Verfassungsentwurf fand in der Konstituanten, in der die Bürgerlichen über eine übermächtige Mehrheit verfügten, keine Zustimmung. Man zog vor, die 1871er-Verfassung als Diskussionsgrundlage heranzuziehen, ohne aber sich im einzelnen den von der sozialdemokratischen Minderheit favorisierten Vorschlägen gänzlich zu verschließen. Dies läßt sich an der Aufnahme des die Sozialgesetzgebung betreffenden Artikeln und dem Verbot der Armee (Art. 12) ablesen.

Insgesamt läßt sich die CP als eine in der Tradition ihrer Vorgängerinnen stehende bürgerlich-demokratische Verfassung charakterisieren, die, grundsätzlich auf dem Präsidialsystem basierend, eine zu dominante Stellung der Exekutive zu verhindern weiß. Als Mittel hierzu wies man insbesondere der rechtssprechenden Gewalt eine starke Kontrollfunktion zu (Wahlgerichtshof-Art. 99-104, Prüfungskompetenz der Gesetzesinitiativen

20 Wie erstaunlich liberal dieses kleine Land mit diesem Problem umgeht ist überraschend, insbesondere wenn man dies mit der um so vieles reicheren Bundesrepublik vergleicht; vgl. dazu: Rojas Franco, Enrique, Dictamen sobre el projecto de ley de asilo político, in: Revista de Ciencias Jurídicas, no. 32, San José 1977 S. $209 \mathrm{ff}$

21 Sandner, Gerhard, Zentralamerika und der Ferne Karibische Westen, Stuttgart 1985, S. 219 f.; vgl. dazu auch: Minkner, Mechthild, Entwicklungsmodell in der Krise Hamburg, 1982. 
auf ihre Verfassungsmäßigkeit-Art. 128). Ferner erwartete man von der Verpflichtung zur zwangsweisen Rotation (Art. 132) einen der Verkrustung von Machtstrukturen, insbesondere der Errichtung einer Herrschaft einer oder weniger Familien (wie etwa in Somozas Nicaragua) entgegenwirkenden Effekt. Eine faktische Omnipotenz der zentralen Exekutivgewalt trachtete man desweiteren durch eine Dezentralisierung der Entscheidungskompetenzen sowohl auf vertikaler wie auch horizontaler Ebene (Kommunale Selbstverwaltung - Art. 168-175; Autonome Einrichtungen - Art. 188-190) zu verhindern. Dem gleichen Ziel diente auch die Verlagerung der Letztentscheidungsbefugnis in Kernfragen auf das Parlament (Art. 121). Wenden wir uns nun den einzelnen Normen der Verfassung zu.

\section{a) Die Republik (Art. 1-2)}

Der erste Abschnitt trifft die den äußeren Rahmen des Staates bestimmenden Grundentscheidungen. Begnügten sich die bisherigen Verfassungen mit einem ausschließlichen Hinweis auf die Souveränität und Unabhängigkeit des Landes, so findet sich seit 1949 auch die Betonung des demokratischen Charakters in der Verfassung (Art. 1). Dies wurzelt in dem Bemühen, sich von den als 'undemokratisch` empfundenen, dem totalitären Kommunismus zuneigend verdächtigten Vorgängerregierungen abzugrenzen. In Art. 6 werden bezugnehmend auf im letzten Jahrhundert mit Nicaragua geschlossene Verträge und dem den Grenzkonflikt mit Panama beilegenden Vertrag von 1941 die Grenzen der Republik festgelegt. 1975 verankerte man außerdem den Anspruch auf die ausschließliche Hoheitsgewalt in der 12-Meilen-Zone und den auf eine besondere Hoheitsgewalt innerhalb der 200-Meilen-Zone, internationalen Gepflogenheiten folgend, in der Verfassung (Ley no 5699 de 5 de junio de 1975). Am selben Tag erlangte der Wahlgerichtshof auch formal (was faktisch schon über Art. 99 ff., das Ley Orgánica del Tribunal Supremo de Elecciones y del Registro Civil und dem Codigo Electoral früher erreicht worden war) seine Gleichstellung mit den klassischen Staatsgewalten (Ley no 5704). Die Ursache dafür liegt in dem Bestreben, ähnliche Vorgänge wie den, der den Bürgerkrieg von 1948 auslöste, zu verhindern.

Der den Abschnitt beschließende Art. 12 ist eine der bemerkenswertesten Normen der Verfassung. Zwar war auch während der Geltungsdauer der '7ler Verfassung - obwohl unter der Regierung eines Generals entstanden - der Einfluß des Militärs auf die Politik zunehmend zurückgegangen, doch stellt der völlige Verzicht auf eine Armee ein Novum dar. Anstoß dafür gab einerseits die Furcht, das Militär könne sich in die Politik mischen, andererseits stellte es ein Resultat einer internen' zweiseitigen Abrüstung dar. Die von den Bürgerlichen geforderte Auflösung der Figueres unterstützenden panzentralamerikanischen 'Legion de Caribe` wurde an die Bedingung geknüpft, daß auch die staatliche Militärmacht kein Machtpotential mehr darstellen dürfe. Trotz dieser politischem Kalkül entspringenden Motivation bleibt der von Costa Rica vorexerzierte Schritt zur Auflösung der Armee eine anerkennenswerte Leistung. Dies umso mehr, wenn man bedenkt, daß das Land bis in die fünfziger Jahre hinein nicht frei von der Ge- 
fahr einer Invasion von Parteigängern Calderóns, bzw. von der somozistischen Nationalgarde war und auch heute sich Mittelamerika als eine äußerst krisengeschüttelte Region präsentiert.

\section{b) Die Costaricaner (Art. 13-18)}

Dieser, Staatsbürgerschaftsfragen betreffende Abschnitt, der sich in jeder der seit 1821 verkündeten Verfassungen findet, regelt dieses Gebiet in größerer Ausführlichkeit als die frühen Konstitutionen. Die Tendenz ist einbürgerungsfreundlich, i. d. R. kann bereits nach fünf Jahren ein Einbürgerungsantrag gestellt werden, sofern dies ein Bürger eines mittelamerikanischen Landes beabsichtigt, reicht ein, bzw. zwei Jahre bei einem Spanier oder einem (sonstigen) Lateinamerikaner Wartezeit aus. Mit dieser einwanderungsfreundlichen Haltung zielte man ursprünglich auf die Behebung des 'Raum ohne VolkProblems`. Heute sollen zusätzlich mit steuerlichen Vorteilen vor allem vermögende USAmerikaner dazu animiert werden, ihren Lebensabend unter Mitnahme ihres Vermögens in Costa Rica zu verbringen, um so der Wirtschaft zu zusätzlichen Investitionsspritzen zu verhelfen. Die ursprünglich vorhandene Neigung ist allerdings angesichts der Krise in den letzten Jahren eher verblaßt.

\section{c) Die Ausländer (Art. 19)}

Ausländer sind Costaricanern prinzipiell gleichgestellt. Sie dürfen sich allerdings seit 1949 nicht mehr, zumindest nicht offiziell, in die Politik des Landes einmischen.

\section{d) Individuelle Rechte und Garantien (Art. 20-49)}

Der erste Teil (Art. 20-33) enthält die klassischen Grundrechte, die auch zum größten Teil in den vorangegangenen Verfassungen Berücksichtigung gefunden hatten. Mit Ausnahme der Art. 33 und 49 wurden seit 1949 auch keine Änderungen mehr vorgenommen. Dies verdeutliche, daß die Entwicklung auf diesem Sektor zu einem gewissen Abschluß gelangt ist. Wie eng diese Artikel an frühere Verfassungen anknüpfen, wird daran deutlich, daß der Abschnitt mit dem expliziten Verbot der Sklaverei (Art. 20) beginnt. Der darauf folgende Artikel verbietet die Todesstrafe. Bereits unter dem 'Vater der 71er Verfassung, Tomás Guardia, nahm man ihn in die Verfassung auf, also zu einem Zeitpunkt, als in den meisten sich als zivilisiert verstehenden Ländern dies noch als zumindest außergewöhnlich, wenn nicht sogar inopportun angesehen wurde. Von der traditionell liberalen Asylpolitik zeugt Art. 31, der allen aus politischen Gründen Verfolgten in Costa Rica Schutz gewährt.

Der zweite Teil dieses Abschnitts betrifft vorwiegend die klassischen strafprozessualen Garantien, als da wären nulla poena sine lege (Art. 39), Verbot von Ausnahmegerichten (Art. 35), Folterverbot (Art. 40) etc. Uber Art. 40 sind auch grausame Strafen untersagt, darunter versteht man in Costa Rica u. a. eine Verurteilung zu lebenslänglicher 
Haft, bzw. die Konfiskation des Vermögens. Die in Lateinamerika weitverbreitete und oft zu Folterungen benutzte incommunidado-Haftı (Kontaktsperre) darf unter keinen Umständen über 10 Tage hinaus ausgedehnt werden und auch während dieser Zeit steht zumindest einem Richter der Zugang zu dem Gefangenen offen (Art. 44). Ergänzt wird der Schutz vor einem 'Verschwinden tragsı nach Art. $48 .{ }^{22}$

Die ideologische Grundhaltung des Verfassungsgebers machen nach Art. 45 und 46 deutlich: Einerseits deklariert man das Eigentum als unverletzlich, andererseits unterwirft man es aber einer Sozialbindung (Enteignungen sind nur zum Wohl der Allgemeinheit gegen Entschädigung zulässig, sofern sich dafür im Parlament eine Zweidrittelmehrheit findet) und sucht die Freiheit von Handel und Gewerbe durch ein Verbot privater Monopole zu schützen.

\section{c) Soziale Rechte und Garantien (Art. 50-74)}

Dieser Abschnitt ähnelt im Hinblick auf seine Kontinuität und Stabilität dem vorangegangenen, wenn er auch erst sehr viel später seine Ausformulierung erfuhr. Seit 1949 reformierte man, abgesehen von redaktionellen Änderungen, keine einzige dieser Normen. Ursprünglich enthielten die Verfassungen keine sozialpolitischen Garantien, sofern man die Schulgeldfreiheit, die die Verfassung von 1869 gewährte, und den allgemein gehaltenen Programmsatz der kurzlebigen Verfassung von 1917 nicht dazuzählen will. Die sozialen Grundrechte entsprechen fast alle nahezu wörtlich den unter Calderón unter Beteiligung der KP verabschiedeten Artikeln. Insoweit hielt Figueres also sein gegebenes Versprechen und auch einer der Verfasser des ursprünglichen Verfassungsentwurfes der Junta, Rodrigo Facio, konzidiert, daß die Kernpunkte des sozialdemokratischen Projekts insoweit in die neue Verfassung Eingang gefunden haben. ${ }^{23}$

$\mathrm{Zu}$ den sozialen Grundrechten zählt auch - ein Zugeständnis an die katholische Kirche, deren Repräsentanten das Bündnis Calderón-KP gefördert hatten - die Unterstellung von Ehe und Familie unter den besonderen Schutz des Staates, aber auch die Sorge für die sozial Schwachen und Unterprivilegierten. Dem abhängig Beschäftigten garantiert Art. 57 einen Mindestlohn, Art. 58 den Achtstundentag und Art. 59 einen Mindesturlaub von zwei Wochen pro 50 Wochen. Den Tarifparteien steht der Weg sowohl in den Streik als auch zur Aussperrung offen (Art. 61). Regulierend greift der Staat ein, wenn er die soziale Situation über die Gründung von Kooperativen (Art. 64), den Bau von Sozialwohnungen (Art. 65), eine Verbesserung der Ausbildungssituation (Art. 67) und den Aufbau eines Sozialversicherungssystems (Art. 73) verbessern helfen will. Diese detaillierte Sozialgesetzgebung, ursprünglich von den Republikanern und der KP unter Mit-

22 Vgl. Mayorga Corea vs. Presidente de la República y Consejo de Gobierno, Corte Plena Ses. ext. No. 61, 10 horas del 10 de noviembre de 1960.

23 In: La Constitución Política de 1949 y la Tendencia Institucional, Revista de la Universidad de Costa Rica, No. 13, San José 1956, S. 98. 
wirkung der katholischen Kirche aus der Taufe gehoben, wurde zu einer der Säulen des Nimbus Costa Ricas. Hinsichtlich Arbeitssituation und Ausbildungswesen ist eine weitgehende Identität von Verfassungsanspruch und -wirklichkeit festzustellen, was die Wohn- und Lebenssituation weiter Teile der Bevölkerung angeht, klafft zwischen diesen noch eine weite Lücke. Die Hoffnung der Prosperitätsperiode auch in diesem Sektor den Durchbruch zu schaffen, hat die Krise seit Mitte der siebziger Jahre allerdings zerschlagen.

\section{f) Die Religion (Art. 75)}

Im einzigen Artikel dieses Abschnitts wird die römisch-katholische Religion langer Verfassungstradition gemäß zur Staatsreligion deklariert, ohne daß die Ausübung anderer Kulte untersagt wird.

\section{g) Die Erziehung und die Kultur (Art. 76-89)}

Der hier geregelte Bereich ist ein weiterer, der zu dem Ruf Costa Ricas, ein Ausnahmefall zu sein, beiträgt. Zwischen 1950 und 1984 konnte mit Hilfe des für lateinamerikanische Verhältnisse gut ausgebauten Schulsystems die Analphabetenquote von fast $21 \%$ auf unter $10 \%$ abgesenkt werden. Für den Pflichtbesuch der Schule muß kein Beitrag entrichtet werden (Art. 78), bedürftige Schüler haben einen Anspruch auf staatliche Stipendien (Art. 82). Parallel zum Bildungsangebot für Jugendliche und Kinder existiert ein breit gefächertes Erwachsenenbildungsprogramm, das über Radio auch schwer zugängliche ländliche Regionen mit einschließt, sowie ein in den letzten Jahren ausgebautes universitäres Bildungsangebot. Welchen Stellenwert man den universitären Einrichtungen beimißt, wird aus der Verfassungsreform von 1975 deutlich. 1949 verfügte das Land über lediglich eine Universität, der über Art. 84 f. und 88 CP ein besonderer Status eingeräumt ist (Autonomie, Ausstattung mit eigenem Vermögen, Beteiligung an die Universität betreffende Gesetzesvorhaben). 1975 stattete man auch die übrigen, inzwischen entstandenen universitären Einrichtungen mit ebendiesen Privilegien aus. ${ }^{24}$

\section{h) Politische Rechte und Pflichten (Art. 93-104)}

Dieser Teil der Verfassung, in dem im wesentlichen das Wahlsystem einschließlich Aufgaben und Zusammensetzung des Obersten Wahlgerichtshofs $(\mathrm{OWGH})$ geregelt werden, zählt zu den am häufigsten geänderten Abschnitten. Behaftet von dem '48er-Trauma einer einen Bürgerkrieg nach sich ziehenden manipulierten Wahl ist man seither stetig bemüht, das Wahlverfahren weiter zu perfektionieren. Dies geht sogar soweit, daß man die Form der Festlegung der Abfolge der Parteien auf dem Wahlzettel wie auch den Mindestabstand zwischen gegnerischen Parteiveranstaltungen im Wahlgesetz normierte, 
obwohl alle Beobachter darin übereinstimmen, daß die costaricanischen Wahlen seit 1949 zu Klagen über 'Unsauberkeiten` keinen Anlaß boten. Ausdruck dieses Traumas ist ferner die Vorschrift des Art. 121 Ziff. 21, wonach Amnestien aller Art möglich sind, nicht aber für Delikte im Zusammenhang mit Wahlen.

Die Kontrolle und Organisation der Wahlen liegt bei dem seit 1975 auch formal als 'Vierte Gewalt` figurierenden OWGH durchgeführt (Art. 99, 102). Er ist ferner befugt, die Wahlen betreffenden Gesetze und Verfassungsvorschriften ausschließlich zu interpretieren, über Beschwerden zu entscheiden und Beamte, die sich unerlaubterweise politisch betätigen, aus ihren Ämtern zu entfernen und ihnen die Fähigkeit zu entziehen, öffentliche Ämter zu bekleiden. Desweiteren untersteht ihm die Polizei (und gegebenenfalls auch das Militär) für die Gewährleistung eines nichtmanipulierten Wahlverlaufs. Seit 1959 herrscht Wahlpflicht (Art. 93), deren Beachtung über das Wahlgesetz, das sogar Haftstrafen vorsieht, durchgesetzt werden soll. Ziel jener Reform war gewesen, die aus Protest den Urnen fernbleibenden Parteigänger Calderóns an dieselben zu zwingen. Diese Maßnahme half zwar, die Wahlbeteiligung im durchschnittlich ca. 20-30 \% zu heben, trotzdem überschreitet sie in der Regel kaum die $80 \%$-Marke.

Ausführliche und insgesamt dreifach geänderte $(1956,1971,1972)$ Regelungen über die Wahlkampfkostenfinanzierung enthält Art. 96. Bestand einst die Ubung, die bei einem Machtwechsel neu eingestellten Beamten (die ihre Stelle zumeist dem ausgeklügelten 'Clientelismo' verdankten) zur Finanzierung heranzuziehen (was unter dem Slogan 'Erstattung der politischen Schulden bekannt war), so ist dies nunmehr explizit verboten. Der Staat beteiligt sich nun an den Wahlkampfkosten, faktisch werden durch entsprechende Zahlungen über 50 Prozent abgedeckt, wobei die Gesamtsumme (maximal 2 Prozent des durchschnittlichen Jahresetats der Republik) proportional zu den Erfolgen der an den Wahlen teilnehmenden Parteien an diese ausgeschüttet wird. Die zunächst auf $10 \%$ festgelegte Sperrklausel senkte man dann auf $5 \%$ ab, damit kleinere Parteien nicht übermäßig benachteiligt werden. Ein Problem ergibt sich daraus, daß die Zahlungen vorab auf der Basis des vorangegangenen Ergebnisses geleistet werden, verliert nun eine Partei überproportional viele Wähler, so kann sich aus der daraus ergebenden Rückzahlungsverpflichtung leicht der finanzielle Ruin derselben ergeben.

1975 reformierte man Art. 98, der die KP (resp. die PVP) von den Wahlen ausgeschlossen hatte und beseitigte so einen Widerspruch zum Anspruch, ein absolut demokratisches Land zu sein. Seither besteht für eine Partei lediglich die Pflicht, sofern sie an Wahlen teilnehmen möchte, in ihrem Programm sich zur Beachtung der Verfassung zu bekennen.

i) Die Legislative (Art. 105-129)

Die legislative Gewalt übt allein der Kongreß aus (Art. 105). Wie bereits in der '7ler Verfassung (Art. 74) verzichtete man auf ein Zweikammersystem, obwohl dies in einigen Vorläufern (zuletzt in der Verfassung von 1917 (Art. 59)) vorgesehen war. Seit 1961 bilden 57, in den Provinzen gemäß deren Bevölkerungsanteil auf jeweils vier Jahre 
(Art. 107) zu wählende Abgeordnete den Kongreß (Art. 106). Die bis dahin geltende Regelung, daß bei Úberschreiten der 1,35 Millionen Grenze je 30000 Stimmberechtigten ein zusätzlicher Deputierter zu wählen ist, gab man auf. In Anbetracht der damals rapiden Bevölkerungszunahmen fürchtete man, durch diese an der Cádiz-Verfassung angelehnte Norm der Úberschaubarkeit des Gremiums verlustig zu gehen. Art. 109 gebietet den Mitgliedern des Parlaments eine penible Abgrenzung zu den anderen Gewalten und vielen Institutionen des Staates. Nicht nur die Mitglieder ersterer sind nicht nur dann nicht wählbar, wenn sie aktuell in ihnen mitwirken, sondern auch wenn sie dies in den letzten sechs Monaten vor der Wahl getan haben. Auch die Leiter der autonomen Einrichtungen, Verwandte des Präsidenten und Spitzenbeamte auf der Provinzebene fallen unter diesen temporär wirksamen Verlust des passiven Wahlrechts, ebenso wie die Abgeordneten der vorangegangenen Wahlperiode (Art. 107). Um einer Verflechtung öffentlicher Aufgaben und privater Interessen vorzubeugen, sind einem Abgeordneten durch die Art. $111 \mathrm{f}$. auch die Ausübung anderer Wahlämter und bestimmte privatwirtschaftliche Aktivitäten (z. B. leitende Funktionen in Betrieben, die mit dem Staat in vertraglichen Bindungen stehen), untersagt, ein Verstoß kann den Verlust des Abgeordnetenstatus nach sich ziehen. In der Praxis verhinderten diese Normen allerdings nur begrenzt den maßgeblichen Einfluß mächtiger Interessengruppen und der sozialen Elite auf das Parlamentsgeschehen. ${ }^{25}$

Außer über die klassischen Parlamentsrechte (z. B. Verabschiedung von Gesetzen Art. 121 Ziff. 1 bzw. des Etats - Art. 121 Ziff. 11) verfügt der Kongreß über folgende Kompetenzen: Von seiner Zustimmung ist die Erlaubnis für fremde Truppen einschließlich Marineeinheiten abhängig, das Territorium zu betreten bzw. in die Häfen einzulaufen (Art. 121 Ziff. 5). Mit Zweidrittelmehrheit ist er in der Lage, im Notstandsfall explizit bezeichnete Grundrechte für bis zu 30 Tagen zu suspendieren (Art. 121 Ziff. 7 und zwar: das Recht auf Freizügigkeit, die Versammlungsfreiheit, die Meinungs- und Pressefreiheit, das 'freedom of information'-Recht und das Recht auf die Einhaltung bestimmter strafprozessualer Vorschriften). Dieselbe Mehrheit ist auch für die Einleitung von Impeachment-Verfahren und die Verkündung von Amnestien erforderlich.

Ausschließlich zuständig ist das Parlament ferner für die Verfügung über der Nation gehörende Güter. Davon ausgeschlossen sind allerdings die nationalen Ressourcen, Eisenbahnen, Häfen und Flugplätze des Landes, die sowohl im Eigentum, wie auch unter der Kontrolle des Staates verbleiben müssen (Art. 121 Ziff. 14). Mit dieser Norm soll verhindert werden, daß sich ein sogenannter 'Staat im Staater bilden kann, wie dies beispielweise die United Company praktiziert hatte. ${ }^{26}$

Im Rahmen des in Art. 123-129 geregelten Gesetzgebungsverfahrens können sowohl die Exekutive wie auch die Judikative entscheidenden Einfluß ausüben. Legt die Exekutive gegen ein Gesetzesvorhaben ihr Veto ein, so bedarf es einer Zweidrittelmehrheit der Par-

25 Vgl. dazu: Arías Sánchez, Oscar, Grupos de presión en Costa Rica, San José 1971; Stone, Samuel, La Dinastia de los Conquistadores, Ciudad Universitaria Rodrigo Facio 1975.

26 Vgl. Anm. 10 oben. 
lamentarier um dieses wieder außer Kraft zu setzen (Art. 127). Die Exekutive ist mittelbar auch in der Lage, den Obersten Gerichtshof zum Zwecke der Uberprüfung eines Gesetzes auf seine Verfassungsmäßigkeit hin anzurufen (Art. 128). Kommt dieser mit einer Zweidrittelmehrheit zu dem Schluß, daß das Gesetz nicht verfassungsmäßig ist, so gilt dieses als verworfen.

\section{j) Die Exekutive}

Die oberste Exekutivgewalt üben der für vier Jahre (Art. 134) zusammen mit zwei Stellvertretern direkt vom Volk gewählte Präsident (Art. 138) und die - ihm unterstehenden - Minister aus (Art. 130). Als Präsident gewählt ist derjenige, der die Mehrheit der Stimmen auf sich vereinigt. Sofern der Sieger des ersten Wahlgangs allerdings nicht mindestens 40 Prozent der Wähler hinter sich bringen kann, findet eine Stichwahl zwischen den beiden Kandidaten mit den besten Ergebnissen statt (Art. 138). Diese Vorschrift will sicherstellen, daß der Gewählte zumindest von einer qualifizierten Minderheit der Stimmbürger unterstützt wird und der Fall ausgeschlossen ist, daß bei vier Kandidaten mit jeweils fast $20 \%$ der fünfte mit etwas über $20 \%$ der Stimmen zum Präsidenten gekürt wird.

War bis 1969, wie auch in der Zeit vor dem Bürgerkrieg, nur die unmittelbare Wiederwahl eines Präsidenten unzulässig, so verschärfte man dann Art. 132 dahingehend, daß ein einmal amtierender Präsident von zukünftigen Wahlen ausgeschlossen bleibt. Diese lex Figueres` zielte darauf ab, dem populären Führer der Aufständischen im Bürgerkrieg und nachmaligen Juntachef und zweimaligen Präsidenten 'Don Peper eine neuerliche Kandidatur nach Ablauf einer zweiten Amtsperiode zu verunmöglichen.

Die Kompetenz des Präsidenten, die aufgrund des in Costa Rica bevorzugten Präsidialmodells umfassender ist, als die eines nicht vom Volk direkt gewählten, sondern von einer ständigen Mehrheit im Parlament abhängigen Regierungschefs, wird von der Verfassung durch mehrere Mechanismen eingeschränkt. Einer dieser ist die Ausstattung des Ministerrates mit eigenen Rechten und Zuständigkeiten. In diesem Rat führt der Präsident den Vorsitz (Art. 147). Zwar verfügt dieser die Befugnis, sowohl die Ernennung als auch die Entlassung von Ministern vorzunehmen (Art. 139 Ziff. 1) und beschränkt sich damit nicht lediglich auf eine Rolle als Primus inter pares, doch verhindert die gemeinsame Aufgabenzuweisung an Präsident und Minister (Art. 140) faktisch eine allzu absolutistische Amtsführung. Sofern sich eine Besetzung eines Ministerpostens als offensichtlicher Fehlgriff herausstellt, so steht dem Kongreß als Korrektiv zu dem Besetzungsrecht des Präsidenten die Eröffnung eines Impeachmentverfahrens gegen diesen Minister zur Verfügung (Art. 121 Ziff. 9 f.). Für Präsidenten wie Minister gelten im übrigen vergleichbare bzw. identische Unvereinbarkeitsregeln wie für Mitglieder der Legislative (Art. 132 bzw. 143). Trotz dieser institutionellen 'Fesseln ' verfügt die Exekutive als 'policy-making-power über die im Vergleich zu den anderen Staatsgewalten stärkste Position. 
Der Oberste Gerichtshof nimmt im judikativen Sektor die führende Stellung ein (Art. 156), seine Mitglieder werden vom Kongreß gewählt (Art. 157) und dies auf zunächst mindestens acht Jahre. Im Gegensatz zu den exekutiven Spitzenpositionen und den Mitgliedern des Parlaments ist ihre Wahlperiode nicht nur auf die doppelte Zeit angelegt, sondern sie bleiben auch von der institutionalisierten 'Zwangsrotation' verschont. Die Verfassung begünstigt sogar den gegenteiligen Effekt: Nur wenn sich mindestens zwei Drittel der Parlamentarier gegen einen Verbleib eines Richters am Obersten Gerichtshof aussprechen, kann dieser von einem anderen ersetzt werden (Art. 158). Auch eine Abwahl während der laufenden Amtsperiode ist nahezu ausgeschlossen, sie setzt die Erhebung einer Anklage gegen einen Richter voraus, bzw. im Falle eines Verstoßes gegen die Disziplinarordnung das Votum von mindestens zwei Drittel seiner Kollegen. (Art. 165). Für die obersten Richter gilt selbstverständlich eine vergleichbar strenge Inkompatibilitätsregelung wie für den Präsidenten, die Minister und die Kongreßabgeordneten (Art. 160 f.). Der Judikative ist damit gleichsam eine Rolle als rruhender Pol im Zusammenspiel der Gewalten zugewiesen, die eine hohe Kontinuität in der Rechtsprechung gewährleisten soll.

Zieht man ein Fazit, so verfügt die Judikative unter der aktuellen Verfassung über die stärkste Stellung in der Geschichte des Landes. Die Zeiten, in der die Einsetzung der obersten Richter dem Präsidenten vorbehalten blieb und sich ihre Amtsdauer danach bestimmte, wie lange sie ihre 'Amtsgeschäfter 'gut‘ ausübten - was natürlich von der Beurteilung des Präsidenten abhing - (vgl. die Verfassung von 1841) gehören eindeutig der Vergangenheit an. Die Position der Judikative wird ferner noch dadurch gestärkt, daß der Kongreß sie vor Verabschiedung eines Gesetzes anhören muß, sofern dieses dieselbe tangiert. Ist der Oberste Gerichtshof in einem solchen Fall der Auffassung, das Gesetz oder ein Teil davon solle nicht in Kraft gesetzt werden, so bedarf es der Zweidrittelmehrheit im Kongreß, um den Obersten Gerichtshof zu rüberstimmen` (Art. 167).

\section{1) Die Gemeindeverfassung (Art. 168-175)}

Die sieben Provinzen Costa Ricas unterteilen sich seit Inkrafttreten des Código Municipal im Jahre $1970^{27}$ in 75 Kantone und diese wiederum in 383 Distrikte (Art. 168). Die Verwaltung der Gemeinden (auf Kantonsebene) unterliegt der Gemeinderegierung (Art. 169). Der Gemeinderat besteht aus fünf bis neun (je nach Größe der Kommune) auf vier Jahre gewählten Gemeinderäten (Art. 171 i. V. m. dem Código Municipal). Ein Exekutivbeamter, dessen Stellung auf den 'Jefe Políticor der Cádiz-Verfassung zurückgeht, verbindet die kommunale mit der zentralstaatlichen Ebene (Art. 169) und kann gegen Beschlüsse des Gemeinderats sein Veto (mit aufschiebender Wirkung) einlegen (Art. 173). Die Einbindung der Distrikte in die kantonale Sphäre wird dadurch herge-

27 Ley No. 4774 de 4 de mayo de 1970. 
stellt, daß ein im Distrikt gewählter 'Sindikus` mit Rede- aber ohne Stimmrecht den Distrikt in der Gemeindeversammlung repräsentiert (Art. 172). Er bildet zusammen mit vier weiteren, von dem Gemeinderat ernannten Bürgern eine Art 'Ortsbeirat', was einer Erhöhung der Bürgernähe dienlich sein soll.

Die Gemeinden besitzen den Status einer juristischen Person, verfügen über ein - begrenztes - Etatrecht (Art. 175) und sind autonom (Art. 170). Uber diese strukturelle Gliederung beabsichtigte man eine als schädlich empfundene übermäßige Machtkonzentration in den Händen des Präsidenten durch eine Dezentralisierung der Entscheidungskompetenzen zu verhindern. Um das Aufkommen eines lokalen Nepotismus zu erschweren, gelten für Gemeinderäte ähnlich strenge Inkompatibilitätsregelungen wie für Parlamentarier (Código Municipal $§ 23 \mathrm{ff}$.).

\section{m) Das staatliche Finanzwesen (Art. 176-187)}

Die ausführliche Reglementierung dieser Materie ist von der Absicht beherrscht, unnötige Staatsausgaben und -verschuldungen, wie sie den Regierungen vor dem Bürgerkrieg zu Last gelegt wurden, zu vermeiden. Bereits der Etatentwurf wird zu diesem Zweck auf exekutiver Ebene von einer zentralisierten Einrichtung geprüft, die das Recht hat, jeden Ansatz, m. A. des den Obersten Wahlgerichtshof betreffenden, zu kürzen oder ganz zu streichen. Eine solche Maßnahme kann dann nur wieder vom Präsidenten rückgängig gemacht werden (Art. 177). Ähnlich wie der OWGH wird auch die Judikative insgesamt vor einer de-facto Ausschaltung geschützt: Mindestens sechs Prozent der jährlichen Staatseinnahmen werden der Judikative zur Verfügung gestellt (Art. 177). Ähnliche Priorität genießt die Sozialversicherungsanstalt.

Als nächstes übt der Kongreß sein traditionelles Etatkontrollrecht aus. Nach Abschluß eines Haushaltsjahres übt dann der Rechnungshof, der unabhängig, aber dem Kongreß als Hilfseinrichtung zugeordnet ist (Art. 183), die Nachkontrolle aus. Auf der Basis eines von diesem erstellten Gutachtens entscheidet dann der Kongreß endgültig. (Art. 181). Eine grundsätzliche Kontrollfunktion räumt Art. 187 auch der Offentlichkeit ein: Alle Ausgaben m. A. der Personalkosten werden grundsätzlich im Staatsanzeiger veröffentlicht (Art. 187). Um die Unabhängigkeit des Rechnungshofes von der Exekutive auch tatsächlich zu garantieren, wählt der Kongreß dessen Leitung auf acht Jahre und ähnlich wie die Mitglieder des Obersten Gerichtshofes genießen dessen Funktionsträger alle Privilegien wie Immunität und Möglichkeit der Wiederwahl (Art. 183).

Trotz dieses anscheinend so engmaschig geknüpften Kontrollnetzes blieb Costa Rica weder grundsätzlich von Finanzskandalen verschont, noch konnte es sich von der allgemeinen Tendenz zur zunehmenden Verschuldung des Staatswesens retten. Nichtsdestotrotz muß den Kontrollmechanismen doch zumindest ein ‘dämpfender Einfluß zugebilligt werden. 


\section{n) Die autonomen Einrichtungen (Art. 188-190)}

Diese Institutionen genießen aus dem gleichen Grund wie die Gemeinden eine relative Autonomie: Der Exekutive soll nur ein begrenzter Kanon ar. Handlungsmöglichkeiten offenstehen. $\mathrm{Zu}$ den wichtigsten traditionellen autonomen Einrichtungen zählen die staatlichen Banken und Versicherungsanstalten (Art. 189), die durch ihr Verhalten aufgrund der ihnen zukommenden wirtschaftlichen Macht natürlich in der Lage sind, die Politik der Regierung entweder zu stützen oder zu konterkarieren.

\section{o) Der öffentliche Dienst (Art. 191-193)}

Um dem Ziel, die staatliche Verwaltung zu effektivieren, gerecht zu werden, stellte die Verfassung die inzwischen erfüllte Aufgabe, ein Gesetz über den öffentlichen Dienst zu erlassen (Art. 191). Art. 192 legt dabei den allgemein verbindlichen Grundsatz fest, daß - um den befürchteten 'Clientelismor zu verhindern - nur die Eignung über eine Einstellung in den öffentlichen Dienst entscheiden darf und eine politisch bedingte Kündigung für die Angehörigen desselben - zumindest theoretisch - nicht vorgenommen werden kann. Die Konsequenz aus dieser Norm besteht in der Durchführung von Eingangs- bzw. Aufnahmeprüfungen bis hinunter auf die kommunale Ebene.

\section{p) Der verfassungsmäßige Eid (Art. 194)}

Der einzige Artikel dieses Abschnitts enthält die Vereidigungsformel, die z. T. wörtlich derjenigen entspricht, die die Abgeordneten der Cortes von Cádiz abzulegen hatten.

\section{q) Verfassungsänderungen (Art. 195-196)}

Abschließend regelt die Verfassung die Vorgehensweise bei einzelne Artikel der Verfassung ändernde Reformen (Art. 195) sowie das Procedere bei einer Totalrevision (Art. 196). Während letztere extra die Wahl und Einberufung einer Verfassungsgebenden Versammlung erfordert, kann eine reinfacher Änderung im Rahmen der rordentlichen 'Tätigkeit des Parlaments vorgenommen werden. Allerdings müssen zwei Drittel der Kongreßmitglieder am Ende eines komplizierten Verfahrens (Antrag von mindestens 10 Abgeordneten, 3 Lesungen, Abstimmung über die Zulassung der weiteren Beratung, Erstellung eines Gutachtens durch einen Parlamentsausschuß, Zweidrittelmehrheit für Weiterbefassung, Úberweisung an einen Redaktionsausschuß, dann an die Exekutive zur Stellungnahme des Präsidenten, weitere drei Lesungen, abschließende Abstimmung) für eine derartige Änderung stimmen.

Bisher verzeichnet man über 30 (erfolgreiche) Initiativen zur Änderung der Verfassung von denen insgesamt über 40 Artikel (einige davon sogar mehrfach) tangiert wurden. 


\section{Fazit}

Bei einer abschießenden Betrachtung kommt man zu dem Schluß, daß die costaricanische Verfassung sich als eir taugliches Instrument im politischen Leben des Landes erwiesen hat. Insbesondere ist zu betonen, daß die politischen Auseinandersetzungen auf dem Boden und unter weitgehender Zustimmung zur Verfassung ausgetragen werden, ein im Vergleich zu nicht wenigen anderen lateinamerikanischen Ländern äußerst bemerkenswerter Umstand. Dem widerspricht auch nicht die relativ häufige Änderung einzelner Artikel. Sie ist vielmehr ein Zeichen von 'Lebendigkeit ‘ und Flexibilität. Ihr ist jedenfalls der Vorzug zu geben vor einer Situation, in der die geschriebene Verfassung zu einer statischen Maske erstarrt, hinter der wie etwa beispielsweise in Chile, Salvador oder Guatemala auch die elementarsten Menschenrechte nur noch auf dem Papier Anerkennung finden.

Verfassungstheoretisch interessant und als Vorbildfunktion sicherlich nicht abzulehnen bleibt die 'Erhöhung' des Obersten Wahlgerichtshofs zur Vierten Gewalt, das Verbot des Militärs in Friedenszeiten sowie die strikte Bemühung um Machtdezentralisation und ständige personelle Neuverteilung der Macht als Gegenmittel gegen eine Verkrustung und Etablierung weniger Familien rauf ewig' an den Schaltstellen des Gemeinwesens. Trotz nicht weniger Kritikpunkte im Detail ist dennoch frappierend, wie relativ geringfügig der Widespruch zwischen Verfassungsanspruch und Verfassungswirklichkeit zumindest auf formalem Sektor ist - eine Feststellung, die von nicht sehr vielen vergleichbaren Ländern für sich in Anspruch genommen werden kann. 
the system is a necessary condition for at least the passive support of the military for the democratic processes. Argentina is in some respects an exception as defeat in the Malvinas/Falklands War of 1982 created an unique situation.

Generally speaking the experiences of dictatorship and exile have led to a reassessment of democracy by Latin American social scientists; the dependence approach of the 1960s has given way to studies on the democratic institutions and the political culture of their countries. However, in view of the precarious economic situation of most of South America the question of whether, despite the present consensus in favour of democracy, democratic government can be consolidated must remain open.

\title{
Costa Rica and its Constitution of 7 November 1949: From the Beginnings to the Present
}

\author{
By Jochen Fuchs
}

The 1949 constitution of Costa Rica is the thirteenth constitutional instrument since the country's declaration of independence from Spain in 1821. During its forty-seven years of existence it has proved a remarkably stable legal foundation of the republic.

Apart from the aspect of constancy this constitution also illustrates the distinctive features of the Costa Rican body politic which have earned the country the epithet of a 'Switzerland of Central Americar.

The author discusses in detail the elements of republicanism and social democracy which have informed the substance of the constitution whose pronounced institutional checks and balances, social basic rights and the proscription of armed forces in peacetime merit particular attention in comparison not only with other Latin American states.

The constitutional order of Costa Rica has from 1949 to the present enjoyed a notably close correspondence of political reality to the legal dispensation of the constitution whereas in many other comparable countries the constitutional framework has atrophied into a mere cloak draped over lawless politics.

\section{The Legal order of Post-Revolutionary Nicaragua: Claims and Reality}

\section{By Marta Patricia Baltodano, Reinhard Junghanns and Michael Sommer}

The basis of this article is the declaration made immediately before the revolution of 19 July 1979 by the Frente Sandinista de Liberacion Nacional (FSLN). In this declaration, the FSLN made a commitment to friendly nations that they would install a democratic form of government, on a foundation of law and order. The first de facto-constitution following the revolution consisted of the Basic Statute (Estatuto Fundamental) enacted 